\title{
Non-Fermi Liquid Behavior Close to a Quantum Critical Point in a Ferromagnetic State without Local Moments
}

\author{
E. Svanidze, ${ }^{1}$ L. Liu, ${ }^{2}$ B. Frandsen, ${ }^{2}$ B. D. White, ${ }^{3}$ T. Besara, ${ }^{4}$ T. Goko, ${ }^{2, \dagger}$ T. Medina, ${ }^{5}$ T. J. S. Munsie, ${ }^{5}$ \\ G. M. Luke, ${ }^{5}$ D. Zheng, ${ }^{6}$ C. Q. Jin, ${ }^{6}$ T. Siegrist, ${ }^{4}$ M. B. Maple, ${ }^{3}$ Y. J. Uemura, ${ }^{2}$ and E. Morosan ${ }^{1, *}$ \\ ${ }^{1}$ Department of Physics and Astronomy, Rice University, Houston, Texas 77005, USA \\ ${ }^{2}$ Department of Physics, Columbia University, New York, New York 10027, USA \\ ${ }^{3}$ Department of Physics, University of California, San Diego, La Jolla, California 92093, USA \\ ${ }^{4}$ National High Magnetic Field Laboratory, Florida State University, Tallahassee, Florida 32306, USA \\ ${ }^{5}$ Department of Physics and Astronomy, McMaster University, Hamilton, Ontario L8S 4M1, Canada \\ ${ }^{6}$ Institute of Physics, Chinese Academy of Sciences, Beijing 100190, China \\ (Received 16 July 2014; revised manuscript received 24 November 2014; published 4 March 2015; corrected 16 March 2015) \\ A quantum critical point (QCP) occurs upon chemical doping of the weak itinerant ferromagnet $\mathrm{Sc}_{3.1} \mathrm{In}$. \\ Remarkable for a system with no local moments, the QCP is accompanied by non-Fermi liquid behavior, \\ manifested in the logarithmic divergence of the specific heat both in the ferro-and the paramagnetic states, \\ as well as linear temperature dependence of the low-temperature resistivity. With doping, critical scaling is \\ observed close to the QCP, as the critical exponents $\delta, \gamma$, and $\beta$ have weak composition dependence, with $\delta$ \\ nearly twice and $\beta$ almost half of their respective mean-field values. The unusually large paramagnetic \\ moment $\mu_{\mathrm{PM}} \sim 1.3 \mu_{B}$ /F.U. is nearly composition independent. Evidence for strong spin fluctuations, \\ accompanying the QCP at $x_{c}=0.035 \pm 0.005$, may be ascribed to the reduced dimensionality of $\mathrm{Sc}_{3.1} \mathrm{In}$, \\ associated with the nearly one-dimensional Sc-In chains.
}

DOI: 10.1103/PhysRevX.5.011026

\section{INTRODUCTION}

Quantum critical points (QCPs) are ubiquitous features in the phase diagrams of strongly correlated electron systems, ranging from high-temperature oxide superconductors [1-3] and low-dimensional compounds [4-6], to itinerant magnets (IMs) [7-10] and heavy fermions (HFs) [11-16]. Often, non-Fermi liquid (NFL) behavior [17-21], and critical scaling [22] accompany the QCP, and such novel phenomena have been extensively studied in HFs and, in particular, in antiferromagnetically ordered systems. However, fewer ferromagnetic QCPs are known and much less is currently understood about itinerant electron magnets and their quantum critical behavior, particularly due to the limited number of existent IMs. These observations make itinerant ferromagnets (IFMs) particularly appealing, given the comparatively smaller number of known ferromagnetic (FM) rather than antiferromagnetic (AFM) QCPs. The QCPs recently observed in two substantively different systems, the IFM $\mathrm{ZrZn}_{2}$ [7] and the HF ferromagnet

\footnotetext{
*Corresponding author. emorosan@ rice.edu

'Present address: Laboratory for Muon Spin Spectroscopy, Paul Scherrer Institut, CH-5232 Villigen PSI, Switzerland.

Published by the American Physical Society under the terms of the Creative Commons Attribution 3.0 License. Further distribution of this work must maintain attribution to the author $(s)$ and the published article's title, journal citation, and DOI.
}

Subject Areas: Condensed Matter Physics

$\mathrm{URu}_{2} \mathrm{Si}_{2}$ [23], are two such FM QCP systems. NFL behavior is associated with the quantum phase transition induced by doping in the latter compound, but not the former, reemphasizing the imperious need for a unified picture of quantum criticality and NFL behavior in IFM systems. This study of the doping-induced NFL state close to the $\mathrm{QCP}$ in the IFM $\mathrm{Sc}_{3.1}$ In provides a first connection between the two previously known ferromagnetic QCP systems, a precursor of such a unified theory.

$\mathrm{Lu}$ doping in $\mathrm{Sc}_{3.1}$ In represents the first report of NFL behavior associated with a QPT in this IFM. The critical composition $x_{c}$ in $\left(\mathrm{Sc}_{1-x} \mathrm{Lu}_{x}\right)_{3.1}$ In is very small, close to 0.035 . The critical scaling close to the QCP is remarkable in comparison to $\mathrm{ZrZn}_{2}$ or $\mathrm{URu}_{2} \mathrm{Si}_{2}$ : While $\mathrm{Sc}_{3.1} \mathrm{In}$ is similar to the former compound as the only other known FM with no magnetic elements, its critical scaling is not mean-field-like (MF-like), akin to that in the latter system. The reduced crystallographic dimensionality of $\mathrm{Sc}_{3.1} \mathrm{In}$, associated with quasi-1D Sc-In chains (inset of Fig. 1), provides one plausible justification for the similarities with the two-dimensional $\mathrm{URu}_{2} \mathrm{Si}_{2}$, in contrast with the three-dimensional $\mathrm{ZrZn}_{2}$. In $\mathrm{URu}_{2} \mathrm{Si}_{2}$, the Kondo effect is inherently coupled with the quantum critical behavior, but $\mathrm{Sc}_{3.1}$ In has no local moments, rendering its magnetism and the QCP even more striking. What makes $\mathrm{Sc}_{3.1}$ In even more unique is the NFL behavior, a trait so far not seen in IFMs without magnetic elements. It is therefore paramount to probe the existence of the QCP in this IFM system, and properly characterize the NFL behavior, as 


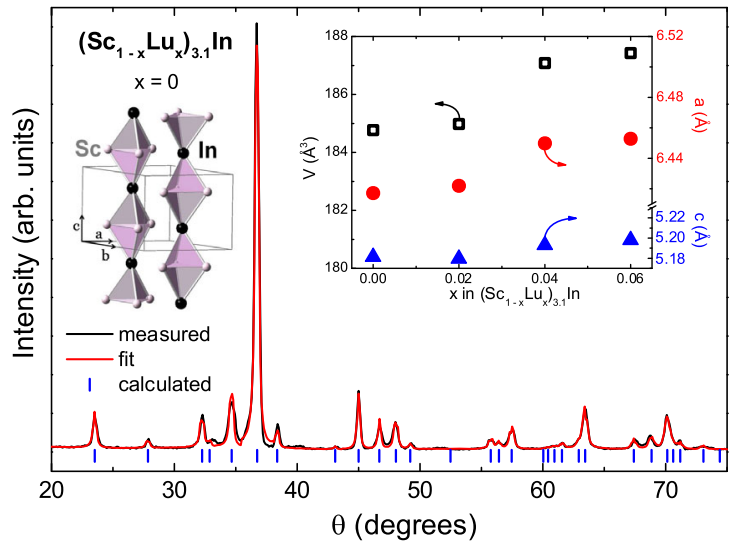

FIG. 1. Measured x-ray diffraction pattern for $\left(\mathrm{Sc}_{1-x} \mathrm{Lu}_{x}\right)_{3.1} \mathrm{In}$ (black line), where $x=0$, with calculated peak positions marked by vertical lines, based on space group $\mathrm{P}_{3} / \mathrm{mmc}$ and lattice parameters $a=6.42 \AA$ and $c=5.18 \AA$. The crystal structure of $\mathrm{Sc}_{3.1}$ In (left inset) exhibits quasi-1D chains of Sc-In. Right inset: Evolution of lattice parameters $a$ and $c$ and the unit-cell volume $V$ with composition $x$.

a precursor for a unified picture of quantum criticality in IFMs. The development of such a unified theory necessitates more IFM systems, which starts with a thorough understanding of the few compounds that are already known.

IFMs lack the complexity associated with the interplay between the local and itinerant character of the electrons observed in HFs $[15,24]$. Of the two known IFMs with no magnetic elements, $\mathrm{ZrZn}_{2}$ and $\mathrm{Sc}_{3} \mathrm{In}$, the latter presents the advantage, from the quantum criticality perspective, of a much lower magnetic ordering temperature $T_{C} \leq 7.5 \mathrm{~K}$ [25-29] in $\mathrm{Sc}_{3} \mathrm{In}$. This would likely facilitate the suppression of magnetic order towards a QCP but has proven difficult by the application of pressure [29] or a magnetic field [27]. Here, we show that the QCP in $\mathrm{Sc}_{3.1} \mathrm{In}$ can indeed be reached by Lu doping, where the dopant ion is comparable in size $\left(r\left[\mathrm{Lu}^{3+}\right]=0.861 \AA\right)$ with the host ion $\mathrm{Sc}$ that it substitutes for $\left(r\left[\mathrm{Sc}^{3+}\right]=0.745 \AA\right)$ [30]. Therefore, the effects of chemical substitution can be deconvoluted from those of chemical pressure, which is important given that pressure was shown to enhance the ordering temperature [29]. The systematic analysis of the magnetization isotherms $M(H)$, temperature-dependent magnetization $M(T)$ at low fields, and $H=0$ specific heat data, for $0 \leq x \leq 0.10$, indicate that the magnetic ground state is suppressed in $\left(\mathrm{Sc}_{1-x} \mathrm{Lu}_{x}\right)_{3.1}$ In towards a QCP close to $x_{c} \approx 0.035$. Remarkably, the logarithmic divergence of the specific heat and the low-temperature resistivity linear in $T$ close to $x_{c}$ are evidence of the NFL behavior in both the ferromagnetic and the paramagnetic states. Additionally, the reduced crystallographic dimensionality, associated with quasi-1D Sc-In chains, may be linked to the NFL behavior and the non-MF critical scaling, similar to that in the more 2D FM, albeit with substantively different critical exponents.

\section{EXPERIMENTAL METHODS}

The hexagonal $\mathrm{Sc}_{3.1}$ In compound has a $\mathrm{Ni}_{3} \mathrm{Sn}$-type structure, with space group $\mathrm{P}_{3} / \mathrm{mmc}$ and lattice parameters $a=6.42 \AA$ and $c=5.18 \AA$ [31]. The reported crystal structure for $\mathrm{Sc}_{3} \mathrm{In}$ is shown in the left inset of Fig. 1 . Highlighted are the Sc-In bipyramids which form nearly one-dimensional chains along the hexagonal $c$ axis. In addition to the nearly one-dimensional crystal structure, band-structure calculations [32] also suggest possible reduced electronic dimensionality. These observations will be discussed in the context of the dimensionality of other IFM systems close to quantum criticality.

It had already been established [25] that $\mathrm{Sc}_{3}$ In forms nonstoichiometrically around the ionic ratio $\mathrm{Sc}: \mathrm{In}=3: 1$. In the current study, we have determined that the optimal composition, which yielded the highest Curie temperature $T_{C}$ and paramagnetic moment $\mu_{\mathrm{PM}}$, was $\mathrm{Sc}: \mathrm{In}=3.1: 1$. Polycrystalline samples of $\left(\mathrm{Sc}_{1-x} \mathrm{Lu}_{x}\right)_{3.1}$ In $(0 \leq x \leq 0.10)$ were prepared by arcmelting Sc (Ames Laboratory, 99.999\%), Lu (Ames Laboratory, 99.999\%) and In (Alfa Aesar, $99.9995 \%$ ), with mass losses of no more than $0.5 \%$. The arcmelted buttons were subsequently wrapped in $\mathrm{Ta}$ foil, sealed in quartz tubes under a partial Ar atmosphere, and annealed over two weeks at temperatures between $700^{\circ} \mathrm{C}$ and $800^{\circ} \mathrm{C}$.

Both annealed and nonannealed samples exhibit extreme hardness, comparable to that of high carbon steel [33], which made it very difficult to perform powder x-ray diffraction measurements. However, it was feasible to $\mathrm{x}$ ray a polished surface of the arcmelted buttons. The arcmelted samples with a radius of about $3 \mathrm{~mm}$ were cut, and the flat surface was scanned for 12 hours in a Rigaku D/Max diffractometer with $\mathrm{CuK} \alpha$ radiation and a graphite monochromator. An example of a diffraction pattern is shown in Fig. 1 for $\left(\mathrm{Sc}_{1-x} \mathrm{Lu}_{x}\right)_{3.1}$ In with $x=0$. All observed peaks can be indexed with the space group $\mathrm{P}_{3} / \mathrm{mmc}$. As shown in the right inset of Fig. 1, both $a$ (right axis, circles) and $c$ (right axis, triangles) lattice parameters, along with the unit-cell volume $V$ (left axis, squares) for $\left(\mathrm{Sc}_{1-x} \mathrm{Lu}_{x}\right)_{3.1} \mathrm{In}$ for $0 \leq x \leq 0.10$, increase nearly linearly with $x$.

The dc magnetization measurements on the annealed samples were performed in a Quantum Design (QD) Magnetic Property Measurement System for temperatures between $1.8 \mathrm{~K}$ and $300 \mathrm{~K}$, and for applied magnetic fields up to $5.5 \mathrm{~T}$. Specific heat was measured from $0.4 \mathrm{~K}$ to $20 \mathrm{~K}$ in a QD Physical Property Measurement System.

Measurements of ac magnetic susceptibility were performed in a ${ }^{4} \mathrm{He}$ dewar down to about $1.17 \mathrm{~K}$, with temperatures below $4.2 \mathrm{~K}$ achieved by pumping on the $\mathrm{He}^{4}$ bath with a Stokes pump. The ac magnetic susceptibility coils were positioned in the thermal gradient above the ${ }^{4} \mathrm{He}$ bath by manually adjusting the vertical position of 
the probe. An ac current was driven on the primary coils with a frequency of $15.9 \mathrm{~Hz}$ using a linear research LR700 ac resistance bridge, which produces an ac magnetic field with a magnitude of around 0.3 Oe. This bridge was also used to measure the in- and out-of-phase components of the signal induced in the secondary pickup coils. The secondary coils are balanced by counterwinding the wire to cancel background signals induced by the oscillating ac magnetic field. A small offset in the measured signal due to minor imbalances in the homebuilt ac susceptibility coils was subtracted from the data. The data were then scaled so that their arbitrary units are proportional to emu/mol.

Muon-spin relaxation $(\mu \mathrm{SR})$ measurements were performed at TRIUMF using a He gas flow cryostat at the M20 beam line for $\mathrm{Sc}_{3.1}$ In and another spectrometer with a dilution cryostat at the M15 beam line for $x=0.01$ and $x=0.025\left(\mathrm{Sc}_{1-x} \mathrm{Lu}_{x}\right)_{3.1}$ In samples. Details of the $\mu \mathrm{SR}$ technique can be found elsewhere [34-38].

\section{RESULTS AND ANALYSIS}

\section{A. Temperature-dependent magnetization measurements}

For weak IFMs, the low-field susceptibility is expected to follow a $T^{-1}$ behavior [39]:

$$
\frac{\chi_{0}}{\chi(T)}=1-\alpha+\lambda(T)
$$

where the coefficient $\lambda$ encompasses the dependence on the local amplitude of spin fluctuations and is linear in temperature $\lambda \sim T / T_{C}^{*}$, and $\alpha=I \rho\left(E_{F}\right)$, where $I$ is the coupling constant and $\rho\left(E_{F}\right)$ is the density of states at the Fermi level. When $T^{*} \gg T_{C}^{*}, \alpha \sim\left(T / T^{*}\right)^{2}$ usually has only a weak $T^{2}$ dependence. However, the magnetic susceptibility $\chi(T)$ in $\mathrm{Sc}_{3.1}$ In follows a modified Curie-Weiss-like law:

$$
\chi(T)=\frac{C}{T^{*}}+\frac{C}{\left(T-T_{C}^{*}\right)},
$$

as illustrated in Fig. 2(a). Such a temperature dependence can possibly be understood when considering strong spin fluctuations, associated with the low-dimensional Fermi surface of $\mathrm{Sc}_{3}$ In [32]: If $T^{*}>T_{C}^{*}$ (but not $\gg T_{C}^{*}$ ), then the temperature dependence of $\alpha$ is not negligible compared to that of $\lambda$. In weak IFMs, the Curie-Weiss-like temperature $T_{C}^{*}$, determined from linear fits of the inverse susceptibility after the temperature-independent term $C / T^{*}$ was subtracted, coincides with the Curie temperature $T_{C}$. As shown below, this is not the case in $\left(\mathrm{Sc}_{1-x} \mathrm{Lu}_{x}\right)_{3.1} \mathrm{In}$, even though $T_{C}$ and $T_{C}^{*}$ are both continuously suppressed to $0 \mathrm{~K}$ with $x$.

A local minimum in the derivative $d M / d T$ [Fig. 2(b), left axis] corresponds to the Curie temperature $T_{C}$ in $\mathrm{Sc}_{3.1}$ In. Moreover, the specific heat data for $x=0$, plotted as $C_{p} / T$ [right axis, Fig. 2(b)], also displays a broad maximum at $T_{C}$. This is remarkable, given that such transitions are often difficult to identify in the fieldindependent properties of IFMs, even in single crystalline samples [23]. In $\mathrm{Sc}_{3.1}$ In, the susceptibility derivatives and specific heat data provide evidence that the ferromagnetic ordering occurs below $T_{C} \sim 4.5 \mathrm{~K}$, as also demonstrated by the field-dependent data shown below. The different measurements consistently indicate that $T_{C}$ is significantly lower than the older estimates from Arrott isotherms alone [26,28,29], when $\mathrm{Sc}_{3}$ In was erroneously assumed to be a MF ferromagnet.

$\operatorname{In}\left(\mathrm{Sc}_{1-x} \mathrm{Lu}_{x}\right)_{3.1} \mathrm{In}, T_{C}$ is continuously suppressed by $\mathrm{Lu}$ doping above $x=0.02$ to values below those accessible by the QD MPMS system. Further data below $T=2 \mathrm{~K}$ were collected from ${ }^{4} \mathrm{He}$ ac susceptibility measurements shown in Fig. 2(c). Lack of data around the $4.2 \mathrm{~K}{ }^{4} \mathrm{He}$ transition precludes a $T_{C}$ estimate for $x=0$ and $x=0.005$, when the transition falls close to this temperature interval. However, for all other compositions up to $x=0.04$, the peak corresponding to $T_{C}$ [illustrated by the solid line fit in Fig. 2(c)] is continuously reduced to temperatures below $T=1.17 \mathrm{~K}$, as shown in Fig. 2(c). This agrees with the critical composition $x_{c}=0.035 \pm 0.005$, as determined from the analysis below.
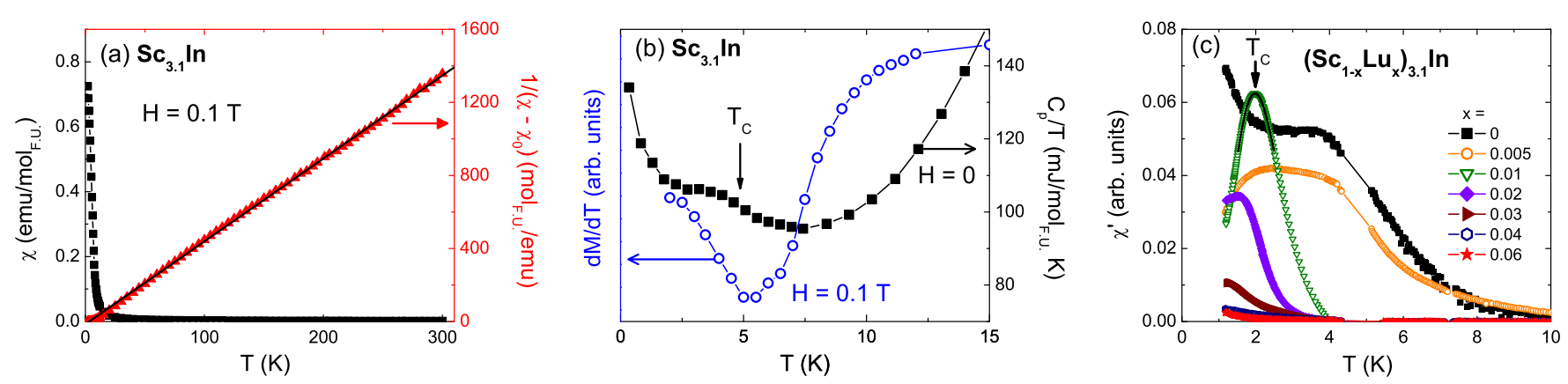

FIG. 2. (a) $\mathrm{Sc}_{3.1}$ In susceptibility (left) and inverse susceptibility $1 /\left(\chi-\chi_{0}\right)$ (right) for $H=0.1 \mathrm{~T}$, where $\chi_{0}=C / T^{*}$ (see text). (b) The magnetization derivative $d M / d T$ (left) and specific heat $C_{p} / T$ (right) for $\mathrm{Sc}_{3.1}$ In with the vertical arrow marking the Curie temperature $T_{C}$. (c) $\left(\mathrm{Sc}_{1-x} \mathrm{Lu}_{x}\right)_{3.1}$ In ac susceptibility $\chi^{\prime}(\mathrm{T})$. The Curie temperature $T_{C}$ is estimated from the peak position (solid line), indicated by the vertical arrow. 

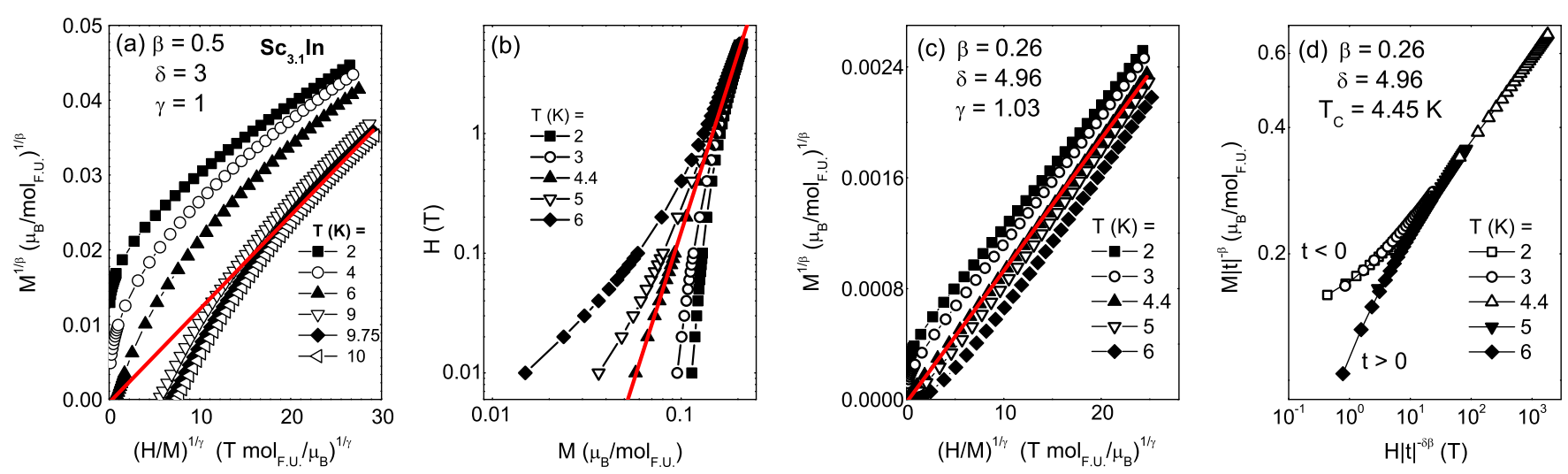

FIG. 3. $\quad M^{1 / \beta}$ vs $(H / M)^{1 / \gamma}$ isotherms for $\mathrm{Sc}_{3.1}$ In with (a) MF exponents $\beta=\beta_{\mathrm{MF}}=0.5$ and $\gamma=\gamma_{\mathrm{MF}}=1, T_{C}=9.75 \mathrm{~K}$ (solid line) and (c) non-MF exponents $\beta=0.26$ and $\gamma=1.03, T_{C}=4.45 \mathrm{~K}$ (solid line). (b) Log-log plot of $\mathrm{Sc}_{3.1} \mathrm{In} \mathrm{M}(\mathrm{H})$ isotherms, with the straight line representing the fit for the critical isotherm. (d) Arrott-Noakes scaling plots $M|t|^{-\beta}$ vs $H|t|^{-\delta \beta}$. The scaled $M(H)$ data collapse onto two diverging branches, one below $T_{C}(t<0$, open symbols $)$ and one above $T_{C}(t>0$, full symbols).

\section{B. Arrott and Arrott-Noakes analysis}

Strong spin fluctuations in $\mathrm{Sc}_{3.1}$ In result in deviations from linearity in the inverse susceptibility around $T_{C}^{*}$ which precludes the accurate determination of the Curie temperature $T_{C}$ from the $\chi(T)$ data. Alternatively, Arrott isotherms $M^{2}$ vs $H / M$ [40] had previously been employed to determine $T_{C}$ in $\mathrm{Sc}_{3} \mathrm{In}$. Existing reports estimate this value as less than $7.5 \mathrm{~K}$ [25-29]. If the Arrott plot technique were used for $\mathrm{Sc}_{3.1}$ In [Fig. 3(a)], it would appear that ferromagnetic order occurred close to $9.75 \mathrm{~K}$. This implies that the Sc-In ratio used for the current study is closest to the optimal one [25], compared to all previous reports. However, the Arrott isotherms deviate strongly from linearity at high $H$ values $[26,28,29]$. This is a compelling indication that the mean-field theory cannot accurately describe the weak ferromagnetism in $\mathrm{Sc}_{3.1} \mathrm{In}$, in contrast with, for example, $\mathrm{ZrZn}_{2}$ [7]. The more generalized Arrott and Noakes method [41] was successfully employed to characterize the critical scaling in the HF ferromagnet $\mathrm{URu}_{2} \mathrm{Si}_{2}$ doped by $\mathrm{Re}$ [23]. In the current work, this generalized critical scaling is applied to a different type of $\mathrm{QCP}$, in the weak IFM $\mathrm{Sc}_{3.1}$ In which has no local-moment elements. It would appear that the NFL behavior results from the non-MF character of the magnetism in these IFMs.

The Arrott-Noakes scaling represents a generalization of the MF scaling of the magnetization $M$, magnetic field $H$ and the reduced temperature $t=\left(T / T_{C}-1\right)$ :

$$
\begin{gathered}
M \propto t^{\beta} \quad \text { for } t<0, \\
M \propto H^{1 / \delta} \quad \text { at } \quad t=0, \\
\chi \propto t^{-\gamma} \quad \text { for } t>0 .
\end{gathered}
$$

This yields generalized critical exponents $\beta, \delta$, and $\gamma$ with the constraint that $1+\gamma / \beta-\delta=0$. In the case of $\left(\mathrm{Sc}_{1-x} \mathrm{Lu}_{x}\right)_{3.1} \mathrm{In}$, the Curie temperature $T_{C}$ and exponent $\delta$ are first determined from $\log \log M(H)$ plots for each composition, as shown in Fig. 3(b) for $x=0$ and in Figs. 4(a)-4(c) for $x=0.005,0.008$, and $x=0.01$. At $T_{C}$, critical scaling requires that the isotherm be linear, with a slope equal to the critical exponent $\delta$. The $T=1.8 \mathrm{~K}$ isotherm for $x=0.02$ is nearly linear all the way down to $H=0 \mathrm{~T}$ [Fig. 4(d)], indicating that $T_{C}$ for $x=0.02$ is finite

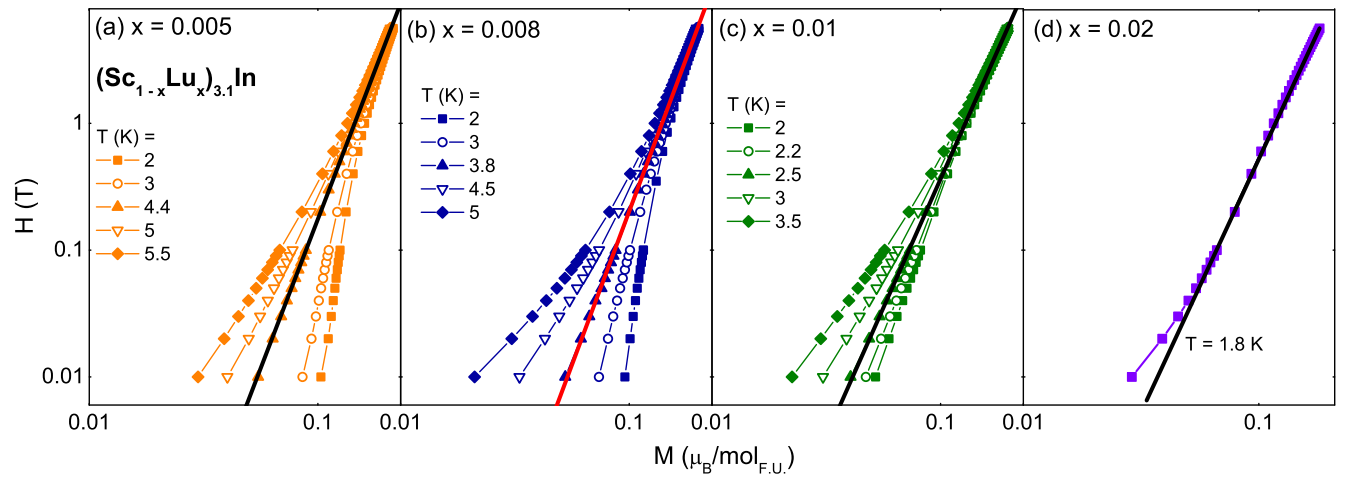

FIG. 4. $\log -\log \left(\mathrm{Sc}_{1-x} \mathrm{Lu}_{x}\right)_{3.1} \mathrm{In} \mathrm{M}(\mathrm{H})$ isotherms for (a) $x=0.005$, (b) $x=0.008$, and (c) $x=0.01$ with linear fits (solid lines) at the critical $\left(T=T_{C}\right)$ isotherm. (d) Log-log $M(H)$ curve for $x=0.02$ and $T=1.8 \mathrm{~K}$, with a linear fit above $H=0.05 \mathrm{~T}$. 

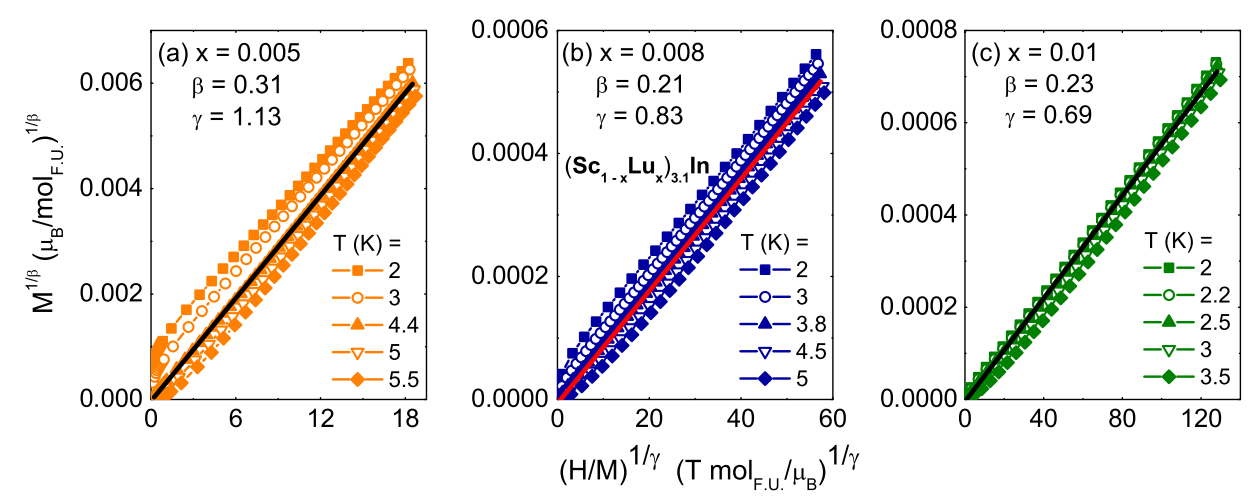

FIG. 5. $\quad\left(\mathrm{Sc}_{1-x} \mathrm{Lu}_{x}\right)_{3.1}$ In Arrott-Noakes $M^{1 / \beta}$ vs $(H / M)^{1 / \gamma}$ isotherms for (a) $x=0.005$, (b) $x=0.008$, and (c) $x=0.01$ with linear fits (solid lines) at $T_{C}$.

and smaller than $1.8 \mathrm{~K}$. For all other FM compositions [Figs. 4(a)-4(c)], nonlinear isotherms occur within $20 \%$ of $T_{C}$. Therefore, in the absence of $M(H)$ measurements below $1.8 \mathrm{~K}$, the nearly linear $\log -\log M(H ; 1.8 \mathrm{~K})$ isotherm for $x=0.02$ is a good indication that the $T_{C}$ value estimate for this composition is within $20 \%$ of $T_{C}$, which yields $T_{C}(x=0.02)=1.5 \pm 0.3 \mathrm{~K}$. This value agrees well with the ac susceptibility estimate, where $T_{C}=1.62 \mathrm{~K}$.

Next, the critical exponents $\beta$ and $\gamma$ are determined from the expected linear dependence of $M^{1 / \beta}$ vs $(H / M)^{1 / \gamma}$. A subset of the resulting isotherms is shown in Figs. 3(c) $(x=0)$ and 5(a)-5(c) $(x=0.005,0.008$ and 0.01). The extrapolations of the linearized isotherms in the ferromagnetic state yield the spontaneous magnetization $M_{0}$ from the vertical axes intercepts. As expected, $M_{0}$ scales with $|t|^{\beta}$, as shown in Fig. 6 for $\left(\mathrm{Sc}_{1-x} \mathrm{Lu}_{x}\right)_{3.1} \mathrm{In}$, where $0 \leq x \leq 0.02$. In contrast with $\mathrm{URu}_{2-x} \mathrm{Re}_{x} \mathrm{Si}_{2}$ [23], $M_{0}$ for $\left(\mathrm{Sc}_{1-x} \mathrm{Lu}_{x}\right)_{3.1}$ In (Fig. 6) grows faster in the ordered state, as the critical exponent $\beta$ for the former, $\beta=0.26 \pm 0.05$, is less than half of the respective value in the latter system [23]. However, the $\beta$ values in $\left(\mathrm{Sc}_{1-x} \mathrm{Lu}_{x}\right)_{3.1}$ In are unusually small, which implies that the ordered moment in this weak IFM is more readily

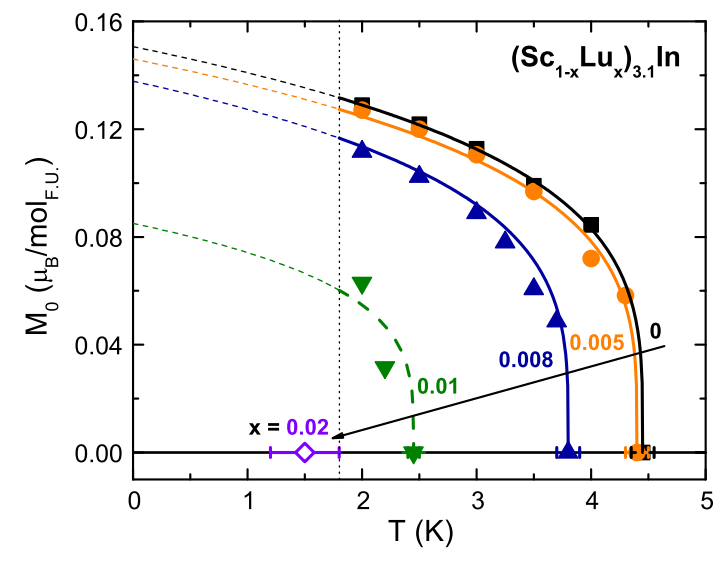

FIG. 6. Spontaneous magnetization $M_{0}(T)$ for $\left(\mathrm{Sc}_{1-x} \mathrm{Lu}_{x}\right)_{3.1} \mathrm{In}$ where $0 \leq x \leq 0.02$. destabilized by fluctuations close to $T_{C}$. This might indicate a fragile magnetism in a nearly $1 \mathrm{D}$ electron system [32], which is immediately suppressed to $0 \mathrm{~K}$ by doping and the attendant disorder.

The Arrott-Noakes critical exponents $\delta$ (triangles), $\gamma$ (squares), and $\beta$ (circles), scaled by their MF values, are presented in Fig. 7 as a function of composition. Most strikingly, $\delta$ is nearly twice as large as its MF value $\delta_{\mathrm{MF}}$, while $\beta$ is nearly half of $\beta_{\mathrm{MF}}$, leaving $\gamma$ nearly identical to its MF value $\gamma_{\mathrm{MF}} . \delta$ is a measure of the curvature of $M(H)$ at $T_{C}$, with larger values signaling faster saturation. A comparison between $\left(\mathrm{Sc}_{1-x} \mathrm{Lu}_{x}\right)_{3.1} \mathrm{In}$ and $\mathrm{URu}_{2-x} \mathrm{Re}_{x} \mathrm{Si}_{2}$ [23] shows that larger $\delta$ values for the former compound are also associated with a larger relative magnetization $M(5.5 \mathrm{~T} ; 1.8 \mathrm{~K}) \approx 0.2 \mu_{B}$. This value at $t=0.6$ is nearly $15 \%$ of the paramagnetic moment $\mu_{\mathrm{PM}} \approx 1.3 \mu_{B}$ for the composition $x=0$ with maximum $T_{C}$. The corresponding value for $\mathrm{URu}_{2-x} \mathrm{Re}_{x} \mathrm{Si}_{2}$ is $M(5.5 \mathrm{~T} ; 1.8 \mathrm{~K}) / \mu_{\mathrm{PM}} \approx$ $\left(0.4 \mu_{B}\right) /\left(3.8 \mu_{B}\right) \approx 10 \%$ (for which $T_{C, \max }=27 \mathrm{~K}$ is obtained for $x=0.6$ ), nearly one-third less at a comparable relative temperature $t$ (Fig. 1, bottom panel, in Ref. [24]).

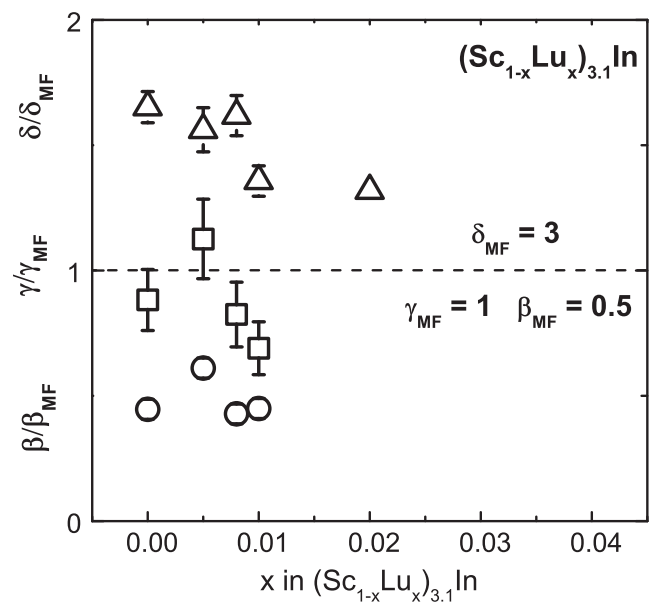

FIG. 7. The critical exponents scaled by their MF values $\delta / \delta_{\mathrm{MF}}$ (triangles), $\gamma / \gamma_{\mathrm{MF}}$ (squares), and $\beta / \beta_{\mathrm{MF}}$ (circles) as a function of composition $x$. 

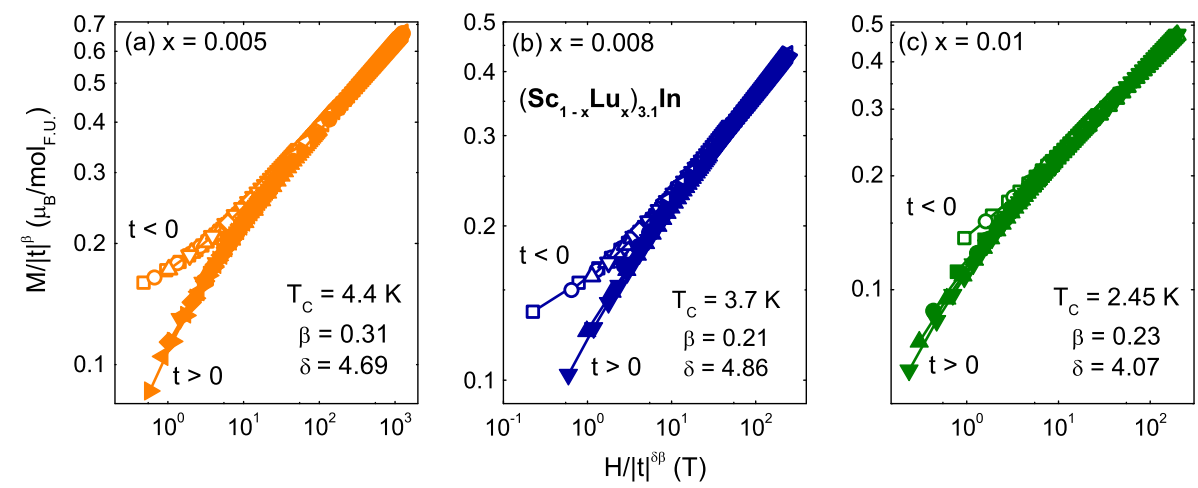

FIG. 8. Scaling plots $M|t|^{-\beta}$ vs $H|t|^{-\delta \beta}$ for (a) $x=0.005$, (b) $x=0.08$, and (c) $x=0.01$ in $\left(\operatorname{Sc}_{1-x} \operatorname{Lu}_{x}\right)_{3.1}$ In.

The scaling collapse of $M|t|^{-\beta}$ vs $H|t|^{-\delta \beta}$, shown in Figs. 3(d) and 8(a)-8(c), exemplifies how the $M|t|^{-\beta}$ vs $H|t|^{-\delta \beta}$ curves collapse onto two diverging branches, for $t<0$ (open symbols) and $t>0$ (full symbols). This collapse is similar to that observed for the $\mathrm{HF} \mathrm{URu}_{2-x} \mathrm{Re}_{x} \mathrm{Si}_{2}$ [23], which is remarkable, given the lack of formal local moments in the constituent elements of $\left(\mathrm{Sc}_{1-x} \mathrm{Lu}_{x}\right)_{3.1} \mathrm{In}$.

\section{Non-Fermi liquid behavior}

Independent and compelling evidence for the QCP in the doped $\mathrm{Sc}_{3.1}$ In system is the NFL behavior below $x=0.04$. One signature of NFL behavior is the logarithmic temperature dependence of the specific heat $C_{p} / T$ [Fig. 9(a)], which occurs over a decade in temperature. The TlnT specific heat, shown in Fig. 9(a), may, in principle, have two possible origins: NFL behavior or Schottky anomaly. For a Schottky anomaly, a low- $T$ peak in the specific heat would move up in temperature with increasing $H$. However, the decrease of the low-temperature $C_{p} / T$ with increasing $H$ [inset, Fig. 9(a)] invalidates the Schottky anomaly scenario, not surprisingly, since this would be associated with low-lying energy states (which is not the case for a system with no formal local moments). The NFL scenario is therefore more plausible in $\left(\mathrm{Sc}_{1-x} \mathrm{Lu}_{x}\right)_{3.1}$ In for $0 \leq x \leq 0.04$. More interestingly, the NFL behavior coexists with the ferromagnetic state. This coexistence has been explained based on magnetic cluster formation as a result of competition between Ruderman-Kittel-Kasuya-Yosida (RKKY) coupling and the Kondo effect $[42,43]$. However, this is the first observation of NFL behavior within the ferromagnetic state in a weak IFM without magnetic elements. The implication is that a new model would be required to describe the ground state in $\mathrm{Sc}_{3.1} \mathrm{In}$, or that the GriffithsMcCoy model [44] may still be appropriate if evidence for the Kondo effect emerges for this compound.

Besides the $T \ln T$ behavior in the specific heat, $\left(\mathrm{Sc}_{1-x} \mathrm{Lu}_{x}\right)_{3.1}$ In displays linear resistivity at the lowest temperatures $\rho(T)=\rho_{0}+A T^{n}(\mathrm{n}=1)$, as shown in Fig. 9(b). A detailed study of the $\left(\mathrm{Sc}_{1-x} \mathrm{Lu}_{x}\right)_{3.1}$ In resistivity and NFL
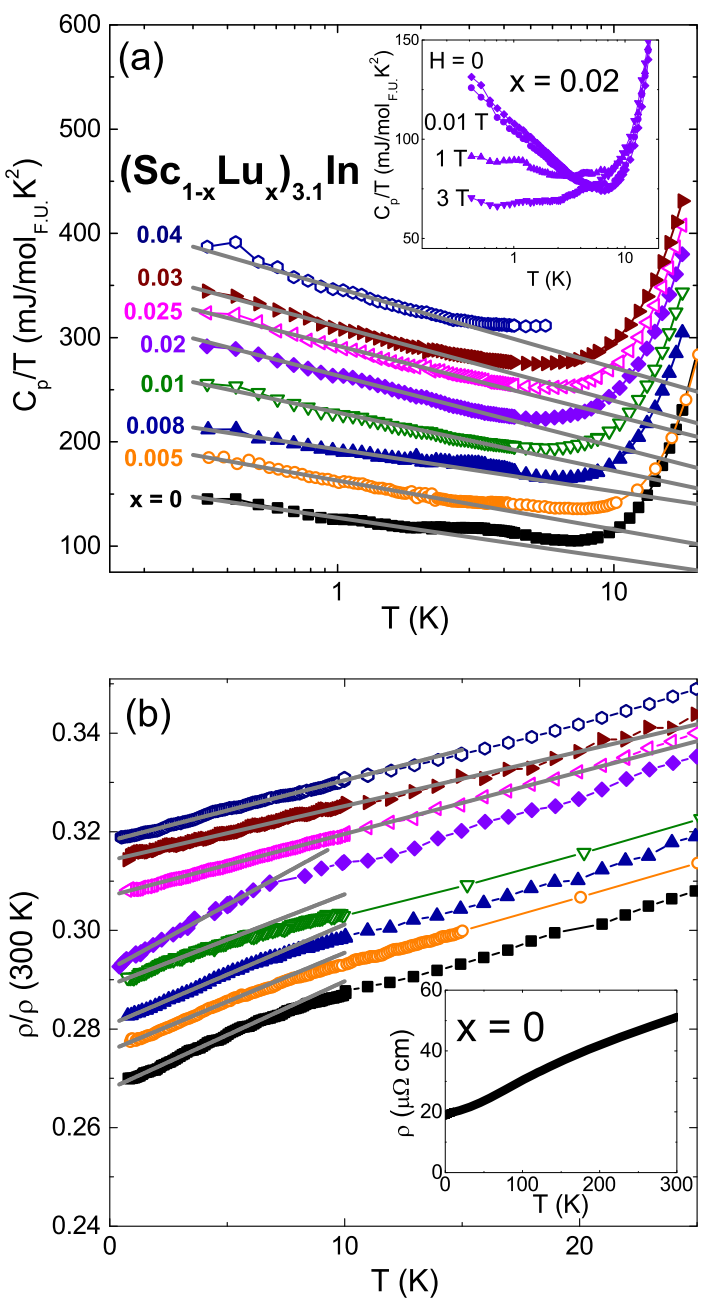

FIG. 9. (a) Semilog plot of the specific heat $C_{p} / T$ (symbols) and the linear fits (lines) at low temperatures. The data for $x>0$ are shifted vertically for clarity by $30 \mathrm{~mJ} / \mathrm{mol}_{\text {F.U. }} \mathrm{K}^{2}$. Inset: Specific heat for $\left(\mathrm{Sc}_{0.98} \mathrm{Lu}_{0.02}\right)_{3.1} \mathrm{In}$ in various magnetic fields: $H=0,0.01,1$, and $3 \mathrm{~T}$. (b) Temperature-dependent resistivity for $\left(\mathrm{Sc}_{1-x} \mathrm{Lu}_{x}\right)_{3.1}$ In $(0 \leq x \leq 0.04)$. The data are shifted vertically for clarity. Inset: The whole range of $\rho(T)$ from which residual resistivity ratios (RRR) are determined. 
TABLE I. Resistivity parameters summary.

\begin{tabular}{lcc}
\hline \hline$x$ & $\rho(0.8 \mathrm{~K})(\mu \Omega \mathrm{cm})$ & $\mathrm{RRR}$ \\
\hline 0 & 19.0 & 2.7 \\
0.005 & 14.7 & 3.5 \\
0.008 & 12.6 & 3.0 \\
0.010 & 15.1 & 2.8 \\
0.020 & 14.6 & 3.1 \\
0.025 & 18.0 & 3.1 \\
0.030 & 15.8 & 2.7 \\
0.040 & 24.1 & 2.7 \\
\hline \hline
\end{tabular}

behavior as a function on both $\mathrm{T}$ and $\mathrm{x}$ is underway [45], revealing that the resistivity exponent remains $n=1$ well beyond $T_{C}$, substantively different from the marginal $\mathrm{FL}$ exponent $n=5 / 3$ of $\mathrm{ZrZn}_{2}$ [46]. Moreover, the specific heat data for $\mathrm{ZrZn}_{2}$ are FL-like [47], with no TlnT dependence like that reported here for $\left(\mathrm{Sc}_{1-x} \mathrm{Lu}_{x}\right)_{3.1} \mathrm{In}$. As illustrated by the inset in Fig. 9(b) for $x=0$, all polycrystalline $\left(\mathrm{Sc}_{1-x} \mathrm{Lu}_{x}\right)_{3.1}$ In samples have comparable $\rho(T)$ values for $0 \leq \mathrm{x} \leq 0.10$. It should be remarked that the absolute residual resistivity values $\rho_{0}$ and the residual resistivity ratios $\mathrm{RRR}=\rho(300 \mathrm{~K}) / \rho_{0} \quad$ (Table I) for $\left(\mathrm{Sc}_{1-x} \mathrm{Lu}_{x}\right)_{3.1}$ In are of the order of $\rho_{0} \sim \mu \Omega \mathrm{cm}$ and RRR around 3-4, values characteristic of good metals, comparable even with those of $\mathrm{ZrZn}_{2}$ single crystals [46]. These are arguments suggesting minimal disorder effects on the quantum critical behavior of the doped $\mathrm{Sc}_{3.1}$ In. Even more important for the question of disorder, the resistivity change $\Delta \rho$ in the range of linearity [Fig. 9(b)] is also comparable to the residual resistivity values $\rho_{0}$, whereas strong disorder effects would result in $\Delta \rho \ll \rho_{0}$. Therefore, the resistivity exponent itself, $n=1$, and the TlnT specific heat in $\left(\mathrm{Sc}_{1-x} \mathrm{Lu}_{x}\right)_{3.1}$ In speak in favor of small disorder effects, in contrast with, for example, doped $\mathrm{Ni}_{x} \mathrm{Pd}_{1-x}$ [48]. In the latter compound, disorder was considered to change the temperature dependence of the specific heat from the expected NFL TlnT in the quantum critical region to $T^{3 / 2}$ slightly away from the QCP. Even for the pressure-induced QCP in MnSi [47], the resistivity exponent is found to be $n=3 / 2$, a value attributed to frozen-in disorder even in very high purity systems.

\section{Muon-spin relaxation measurements}

The small saturation moments of itinerant systems preclude the accurate determination of the small ordered moment from neutron diffraction investigations, similar to the case of $\mathrm{ZrZn}_{2}$ [49]. On the contrary, the muon-spin relaxation $(\mu \mathrm{SR})$ technique is extremely sensitive to local magnetic fields and has been used to investigate multiple itinerant systems [50-54]. Figure 10 shows the time spectra observed in the zero field (ZF) and longitudinal field (LF) at the lowest temperature. The fast relaxation in the early time region in ZF is eliminated by the decoupling effect in LF, which indicates that the observed relaxation

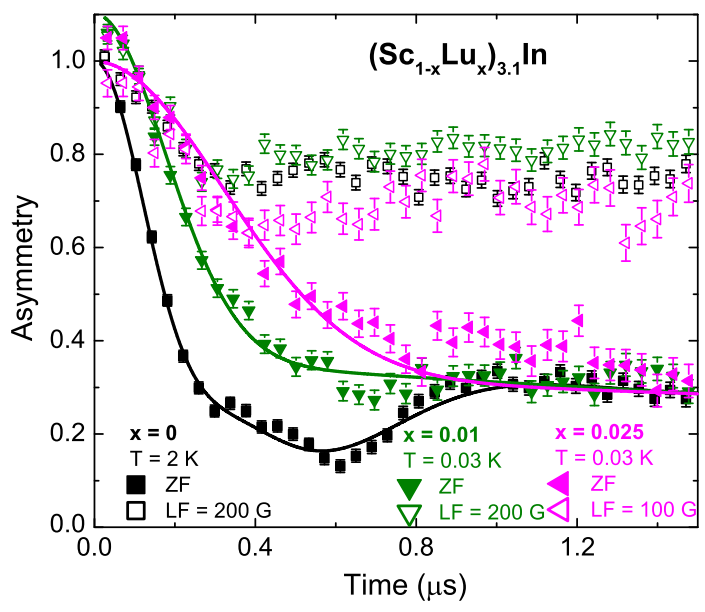

FIG. 10. Time spectra of ZF and LF $\mu$ SR of $\left(\mathrm{Sc}_{1-x} \mathrm{Lu}_{x}\right)_{3.1} \mathrm{In}$, where $x=0$ (squares), 0.01 (downward-facing triangles), and 0.025 (leftward-facing triangles). The background sample holder contribution in the two Lu-doped samples was subtracted. The solid lines represent fits to Eqs. (6) and (7).

is due to a static field, generated by the static magnetic order in both the undoped and doped systems. For the ZF $\mathrm{Sc}_{3.1}$ In spectrum, two precession frequencies at low temperatures can be seen, as shown in Fig. 11(a). The ZF time spectra were analyzed by assuming a functional form of

$$
\begin{aligned}
G(t)= & {\left[A_{1} \cos \left(2 \pi \nu_{1} t\right) e^{\left[-\Lambda_{1}^{2}\left(t^{2} / 2\right)\right]}+A_{2} \cos \left(2 \pi \nu_{2} t\right) e^{\left[-\Lambda_{2}^{2}\left(t^{2} / 2\right)\right]}\right.} \\
& \left.+\left(A_{1 Z}+A_{2 Z}\right) e^{\left[-\left(t / T_{1}\right)\right]}\right] V_{M} \\
& +\frac{\left(1-V_{M}\right)\left[G_{\mathrm{KT}}\left(t, \Delta_{\mathrm{KT} 1}\right)+G_{\mathrm{KT}}\left(t, \Delta_{\mathrm{KT} 2}\right)\right]}{2}
\end{aligned}
$$

where $G_{\mathrm{KT}}(t)$ is the Kubo-Toyabe function [55] for random nuclear dipolar fields, and $A_{1 Z}$ and $A_{2 Z}$ are assumed to be half of $A_{1}$ and $A_{2}$, respectively, as expected for polycrystalline specimens. A very good fit was obtained by assuming $A_{1}=A_{2}$, presumably due to two magnetically nonequivalent muon sites populated with equal probabilities. For the longitudinal relaxation rate $1 / T_{1}$, two values for the two different sites could not be resolved. So, one value of $T_{1}$ was used in the fit. The temperature dependence of the two frequencies $\nu_{1}$ and $\nu_{2}$ is shown in Fig. 11(a). The volume fraction $V_{M}$ of the magnetically ordered region, shown in Fig. 11(b), was determined from the amplitudes of the precession signals. The volume fraction $V_{M}$ decreases gradually with increasing temperature, indicating coexistence of volumes (or regions) with and without static magnetic order. Although the precession signal disappears around $T=5.5 \mathrm{~K}$, a small $V_{M}$ remains above this temperature up to $T \approx 8 \mathrm{~K}$. This is due to a nonprecessing but relaxing signal with a small amplitude, caused by static random fields from the electron system remaining in a small volume fraction. 

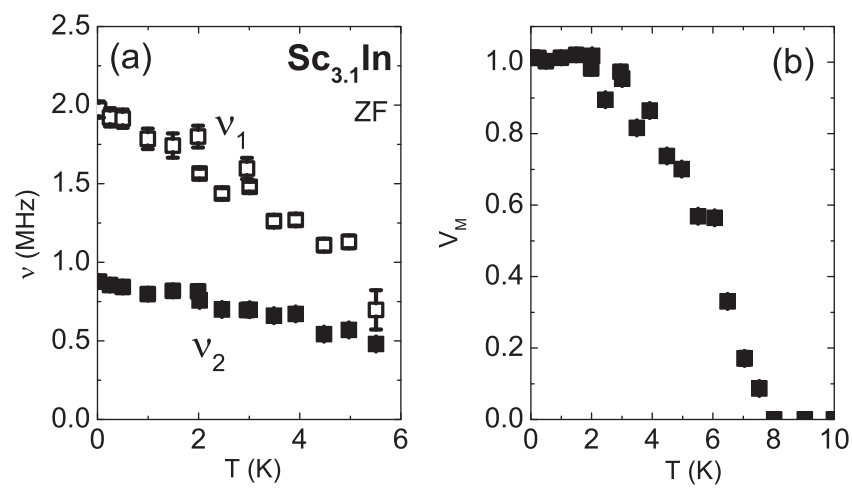

FIG. 11. (a) The muon-spin precession frequencies $\nu_{1}$ (full squares) and $\nu_{2}$ (open squares), and (b) the volume fraction $V_{M}$ of the magnetically ordered regions, obtained from $\mathrm{ZF} \mu \mathrm{SR}$ of $\mathrm{Sc}_{3.1} \mathrm{In}$.

The Lu-doped samples show a relaxing signal without precession at low temperatures, indicating a more random internal field, as compared with the undoped $\mathrm{Sc}_{3.1} \mathrm{In}$. In order to reproduce the observed line shape, the ZF time spectra of the Lu-doped samples have been analyzed by assuming the following functional form:

$$
G(t)=A_{1}\left(1-p \sigma^{2}\right) e^{-\frac{1}{2} \sigma^{2} t^{2}}+\frac{A_{1}}{2} e^{\left[-\left(t / T_{1}\right)\right]}+A_{\mathrm{bg}},
$$

where the first term represents the transverse relaxation, the second term is the longitudinal $1 / T_{1}$ component, and the third term is a background signal from the sample holder. From independent measurements in a weak transverse field at low temperatures, the values of the nonrelaxing background signal from a silver sample holder $A_{b g}$ were estimated to be 0.36 and 0.55 for $x=0.01$ and 0.025 samples, respectively. These values are consistent with the known background level from the cryostat and sample holder, and a rather small sample size. However, it is not possible to eliminate the possibility that a signal from a small paramagnetic volume in the specimen, persisting to $T=0 \mathrm{~K}$, is included in the background signal. Because of the difficulty in separating the effects of slow relaxation and partial volume fraction, the amplitude $A_{1}$ was fixed to be temperature independent, allowing us to extract the relaxation rate $\sigma$. A phenomenological "dip" parameter $p$ $(p=1)$ for the Kubo-Toyabe function was introduced, while smaller $p$ values would fit line shapes with a shallower dip, which are often observed in real materials, including the present case of Lu-doped systems. Although the fit is not perfect, as shown by the lines in Fig. 10, the functional form of Eq. (7) was used to compare the relaxation rates in different specimens without introducing additional free parameters. Figure 12(a) shows the temperature dependence of $\sigma$ in the two Lu-doped specimens. To compare the relaxation rates $\sigma$ with the static field measured in undoped $\mathrm{Sc}_{3.1} \mathrm{In}$, the spatially averaged value of the static local field was determined as
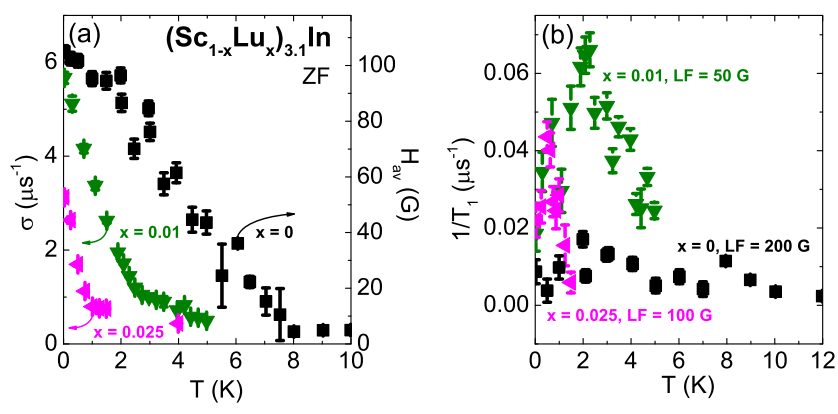

FIG. 12. (a) Muon-spin relaxation rate $\sigma$ for $x=0.01$ (downward-facing triangles) and $x=0.025$ (leftward-facing triangles) for $\left(\mathrm{Sc}_{1-x} \mathrm{Lu}_{x}\right)_{3.1}$ In (left axis), along with the average static internal field $H_{\text {av }}$ for $x=0$ (right axis), obtained from the fits of ZF $\mu$ SR measurements. The vertical axes are scaled with $\gamma_{\mu}$, the gyromagnetic ratio of a positive muon. (b) The longitudinal relaxation rate $1 / T_{1}$, obtained from the $\mathrm{LF} \mu \mathrm{SR}$ measurements.

$H_{\mathrm{av}}=V_{M} \frac{A_{1} \nu_{1}+A_{2} \nu_{2}}{A_{1}+A_{2}}+\frac{\left(1-V_{M}\right)\left(\Delta_{\mathrm{KT} 1}+\Delta_{\mathrm{KT} 2}\right)}{2 \gamma_{\mu}}$,

where $\gamma_{\mu}$ represents the gyromagnetic ratio of a positive muon and $\Delta_{\mathrm{KT}}$ are the widths of the Kubo-Toyabe function for nuclear dipolar fields. We plot $H_{\mathrm{av}}$ in Fig. 12(a) with the relaxation rate (left axis) and the average field (right axis), scaled with $\gamma_{\mu}$. Since the static internal field is expected to be proportional to the local static spin polarization, Fig. 12(a) demonstrates the development of the spatially averaged magnetic order parameter which can be compared to the spontaneous magnetization $M_{0}$, shown in Fig. 6 .

Measurements of the spin-lattice relaxation rate $1 / T_{1}$ were performed in an applied LF. Figure 12(b) shows the temperature dependence of $1 / T_{1}$ for $\left(\mathrm{Sc}_{1-x} \mathrm{Lu}_{x}\right)_{3.1} \mathrm{In}$ with $x=0,0.01,0025$. There is no divergent behavior in $\mathrm{Sc}_{3.1} \mathrm{In}$, while the Lu-doped samples exhibit a peak in $1 / T_{1}$ at the ordering temperature. In either case, the absolute values of $1 / T_{1}$ are less than $0.1 / \mu \mathrm{s}$, which implies that the relaxation rate measured in the zero field [Fig. 12(a)] is predominantly due to a static field, even at temperatures very close to the ordering temperature. In Fig. 12(a), a finite relaxation rate or average field persists up to high temperatures for all three systems. This is attributed to the nuclear dipolar field, as Sc has a very large nuclear moment.

The absence of dynamic critical behavior and the gradual change of the volume fraction $V_{M}$, observed in undoped $\mathrm{Sc}_{3.1}$ In, indicate a first-order transition at the magnetic order. It is interesting to note that a weak "second-order" feature is observed for Lu-doped samples. However, further experimental data are needed given the fact that the order parameter [Fig. 12(a)] exhibits a nonlinear dependence on the Curie temperature $T_{C}$, suggesting a remaining effect of first-order quantum evolution. Additionally, the difficulty in separating the effects of moment sizes and volume fraction at very small relaxation rates, as well as the 
uncertainty in estimating the background level, prevents a reliable determination of $V_{M}$ for the Lu-doped samples.

\section{DISCUSSION}

The paramagnetic moment $\mu_{\mathrm{PM}}$ [diamonds, Fig. 13(a)], determined by the Curie-Weiss-like law, is nearly composition-independent $\mu_{\mathrm{PM}} \sim 1.3 \mu_{B} /$ F.U. Moreover, the Weiss-like temperature $T_{C}^{*}$ decreases nearly linearly with $x$ for $x \leq 0.10$, after an initial jump between $x=0$ and 0.005 [squares, Fig. 13(a)]. Considering that CurieWeiss-like behavior in the itinerant scenario arises from the temperature dependence of the amplitude of spin fluctuations [39], this sudden increase in the corresponding $T_{C}^{*}$ signals enhanced spin fluctuations due to the disorder brought on by Lu doping. Between $x=0.02$ and $x=0.04$, $T_{C}^{*}$ changes sign in a continuous manner, suggesting the presence of a (second-order) doping-induced QCP in this composition range near $x_{c}=0.035 \pm 0.005$. Moreover, $T_{C}$ determined either from $\chi^{\prime}(T)$ or $M(H)$ data [Figs. 2(c) and 5] moves down continuously in temperature below $1.17 \mathrm{~K}$ for the doping amounts above 0.03 , indicating that the QCP is close to this composition.

The determination of the critical composition $x_{c}$ at the QCP requires consistency between the $M(T)$ and $\chi^{\prime}(T)$ data, the critical scaling analysis of the $M(H)$ measurements as well as the $\mu \mathrm{SR}$ results. Indeed, the critical composition $x_{c}=0.035 \pm 0.005$ is determined from (i) the $T_{C}$ (circles and triangles) and $T_{C}^{*}$ (squares) values [Fig. 13(b)] approaching $0 \mathrm{~K}$ at the QCP and (ii) the critical scaling rendering the Arrott-Noakes plots $M^{1 / \beta}$ vs $(H / M)^{1 / \gamma}$ as parallel isotherms, equally spaced in $t$ [Figs. 3(c) and 5(a)-5(c)]. Moreover, the $\mu$ SR results confirm the development of static magnetic order with a nearly full volume fraction at low temperatures and a diminishing moment size as a function of decreasing ordering temperature. Moreover, the continuous variation of $T_{C}^{*}$ and $T_{C}$ with $x$ and the $\mu$ SR evidence for a secondorder phase transition in the Lu-doped samples are also evidence for the QPT induced by Lu doping.

Doping in $\mathrm{Sc}_{3.1}$ In reveals intriguing traits associated with quantum criticality in general and with weak IFM systems in particular: The paramagnetic moment $\mu_{\mathrm{PM}}$ is surprisingly large in $\left(\mathrm{Sc}_{1-x} \mathrm{Lu}_{x}\right)_{3.1}$ In and nearly independent of $x$, even as the system goes through the QPT at $x_{c}=0.035 \pm 0.005$. Not surprisingly then, the critical exponent $\beta$ is unchanged through the ferromagnetic state, although its value $\beta=0.26 \pm 0.05$ is smaller than that in any other known quantum critical system. The minute critical composition and small $\beta$ value, together with the jump in $T_{C}^{*}$ as $x>0$ [Fig. 13(b), squares], point to a weak IFM ground state, easily perturbed by doping. This may seem unusual in light of the stark differences between $\mathrm{Sc}_{3.1}$ In and the related IFM system $\mathrm{ZrZn}_{2}$ [7], with MF specific heat and marginal FL resistivity behavior, or the similarities with the extraordinary critical scaling in the HF FM URu ${ }_{2-x} \mathrm{Re}_{x} \mathrm{Si}_{2}$ [23], close to these systems' respective doping-induced QCPs. However, these observations may be reconciled from crystallographic and electronic property considerations: As a nearly 1D structure is formed by bipyramidal Sc-In chains (inset of Fig. 1), the reduced dimensionality in $\mathrm{Sc}_{3.1}$ In renders it more similar to the layered (2D) $\mathrm{URu}_{2} \mathrm{Si}_{2}$ compound than the cubic (3D) $\mathrm{ZrZn}_{2}$. It appears that the NFL behavior in the ferromagnetic state may also be correlated with the non-MF scaling and, more importantly, that this correlation is independent of the presence of hybridized $f$ electrons. Consequently, the universality of the quantum critical behavior common to the former two compounds may be ascribed to spin fluctuations, associated with reduced crystallographic dimensionality. More IFM systems are needed to probe this universality. Equally important is the synthesis of single crystals of $\mathrm{Sc}_{3.1} \mathrm{In}$,

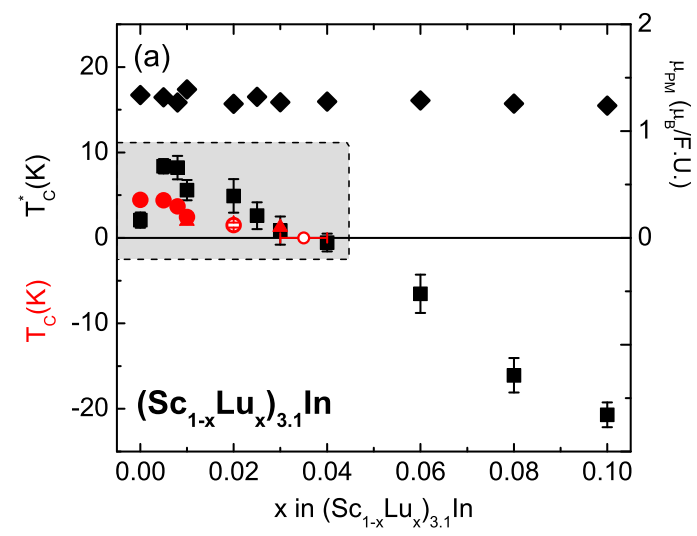

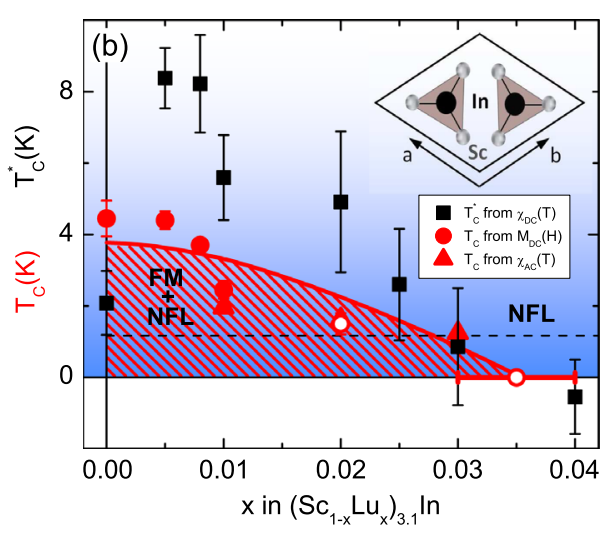

FIG. 13. (a) $T-x$ phase diagram for $\left(\mathrm{Sc}_{1-x} \mathrm{Lu}_{x}\right)_{3.1}$ In for $0 \leq x \leq 0.10$, with the Weiss-like temperature $T_{C}^{*}$ (squares, left axis), the Curie temperature $T_{C}$ (circles, left axis) and paramagnetic moment $\mu_{\mathrm{PM}}$ (diamonds, right axis). (b) Enlarged $T-x$ phase diagram for $x \leq 0.04$ [shaded area in (a)], with the $a b$ plane projection of the $\mathrm{Sc}_{3.1}$ In unit cell shown in the inset. The horizontal line at $T=1.17 \mathrm{~K}$ denotes the minimum experimental temperature, with the open symbols representing $T_{C}$ estimates extrapolated from accessible measurements. 
which would enable further characterization of the implications of dimensionality on the QCP, as well as to probe the potential NFL behavior at the QCP and in the ferromagnetic state. These experiments are currently underway.

\section{ACKNOWLEDGMENTS}

We thank L. L. Zhao for help with figure edits, P.C. Canfield for providing some of the rare-earth metals, and Q. Si and M. C. Aronson for useful discussions. The work at Rice University was supported by National Science Foundation Grant No. DMR 0847681. Measurements of the AC magnetic susceptibility were performed at UCSD with support from the National Science Foundation under Grant No. DMR-1206553. Work at Columbia and TRIUMF (L. L., B.F., and Y.J. U.) was supported by NSF Grant No. DMR-1105961, DMREF Project No. NSF-DMR-1436095, and Grant No. OISE-0968226 (PIRE), a REIMEI project from JAEA, Japan, and the Friends of Todai Inc. Foundation. Work at IOPCAS was supported by NSF and MOST of China through research projects. T. B. and T.S. acknowledge support by the Department of Energy, Basic Energy Science, Division of Materials Science under award DE-SC0008832, and the National High Magnetic Field Laboratory, which is supported by National Science Foundation Cooperative Agreement No. DMR-1157490 and the State of Florida.

[1] J. Orenstein and A. J. Millis, Advances in the Physics of High-Temperature Superconductivity, Science 288, 468 (2000).

[2] S. Sachdev, Quantum Criticality: Competing Ground States in Low Dimensions, Science 288, 475 (2000).

[3] G. S. Boebinger, Y. Ando, A. Passner, T. Kimura, M. Okuya, J. Shimoyama, K. Kishio, K. Tamasaku, N. Ichikawa, and S. Uchida, Insulator-to-Metal Crossover in the Normal State of $\mathrm{La}_{2-x} \mathrm{Sr}_{x} \mathrm{CuO}_{4}$, Phys. Rev. Lett. 77, 5417 (1996).

[4] Y. F. Dai, H. Zhang, S. Y. Zhou, B. Y. Pan, X. Qiu, X. C. Hong, T. Y. Guan, J. K. Dong, Y. Chen, and S. Y. Li, Unveiling the Quantum Critical Point of an Ising Chain, arXiv:1103.0095v1.

[5] D. J. W. Geldart and D. Neilson, Quantum Critical Behavior in the Insulating Region of the Two-Dimensional MetalInsulator Transition, Phys. Rev. B 76, 193304 (2007).

[6] I. Hetel, T. R. Lemberger, and M. Randeria, Quantum Critical Behavior in the Superfluid Density of Strongly Underdoped Ultrathin Copper Oxide Films, Nat. Phys. 3, 700 (2007).

[7] D. A. Sokolov, M. C. Aronson, W. Gannon, and Z. Fisk, Critical Phenomena and the Quantum Critical Point of Ferromagnetic $\mathrm{Zr}_{1-x} \mathrm{Nb}_{x} \mathrm{Zn}_{2}$, Phys. Rev. Lett. 96, 116404 (2006).

[8] A. Subedi and D. J. Singh, Band Structure and the Itinerant Magnetism in Quantum Critical $\mathrm{NbFe}_{2}$, Phys. Rev. B 81, 024422 (2010).
[9] H. Kadowaki, Y. Tabata, M. Sato, N. Aso, S. Raymond, and S. Kawarazaki, Quantum Critical Point of Itinerant Antiferromagnet in the Heavy Fermion, Phys. Rev. Lett. 96, 016401 (2006).

[10] M. Yamada and S. Tanda, Quantum Critical Behavior in Itinerant Ferromagnet $\mathrm{Pd}_{1-x} \mathrm{Ni}_{x}$, Physica (Amsterdam) 284B, 1305 (2000).

[11] A. Schroder, G. Aeppli, R. Coldea, M. Adams, O. Stockert, H. v. Löhneysen, E. Bucher, R. Ramazashvili, and P. Coleman, Onset of Antiferromagnetism in Heavy-Fermion Metals, Nature (London) 407, 351 (2000).

[12] O. Trovarelli, C. Geibel, S. Mederle, C. Langhammer, F. M. Grosche, P. Gegenwart, M. Lang, G. Sparn, and F. Steglich, $\mathrm{YbRh}_{2} \mathrm{Si}_{2}$ : Pronounced Non-Fermi-Liquid Effects above a Low-Lying Magnetic Phase Transition, Phys. Rev. Lett. 85, 626 (2000).

[13] S. L. Bud'ko and P. C. Canfield, Field-Induced Quantum Criticality in YbAgGe, Physica (Amsterdam) 403B, 1230 (2008).

[14] S. Doniach, The Kondo Lattice and Weak Antiferromagnetism, Physica (Amsterdam) 91B+C, 231 (1977).

[15] G. R. Stewart, Non-Fermi-Liquid Behavior in $d$ - and f-electron Metals, Rev. Mod. Phys. 73, 797 (2001).

[16] T. Moriya, Recent Developments in the Theory of Spin Fluctuations in Itinerant Electron Magnets, Physica (Amsterdam) 86B+C, 356 (1977).

[17] A. J. Millis, Effect of Nonzero Temperature on Quantum Critical Points in Itinerant Fermion Systems, Phys. Rev. B 48, 7183 (1993).

[18] M. Tsvelik and M. Reizer, Phenomenological Theory of Non-Fermi-Liquid Heavy-Fermion Alloys, Phys. Rev. B 48, 9887 (1993).

[19] P. Gegenwart, Q. Si, and F. Steglich, Quantum Criticality in Heavy-Fermion Metals, Nat. Phys. 4, 186 (2008).

[20] M. Continentino, On the Zero Temperature Critical Point in Heavy Fermions, Z. Phys. B 101, 197 (1996).

[21] P. Coleman, Theories of Non-Fermi Liquid Behavior in Heavy Fermions, Physica (Amsterdam) 259B, 353 (1999).

[22] W. Knafo, S. Raymond, J. Flouquet, B. Fak, M. A. Adams, P. Haen, F. Lapierre, S. Yates, and P. Lejay, Anomalous Scaling Behavior of the Dynamical Spin Susceptibility of $\mathrm{Ce}_{0.925} \mathrm{La}_{0.075} \mathrm{Ru}_{2} \mathrm{Si}_{2}$, Phys. Rev. B 70, 174401 (2004).

[23] N. P. Butch and M. B. Maple, Evolution of Critical Scaling Behavior near a Ferromagnetic Quantum Phase Transition, Phys. Rev. Lett. 103, 076404 (2009).

[24] A. Aguayo and D. J. Singh, Itinerant Ferromagnetism and Quantum Criticality in $\mathrm{Sc}_{3} \mathrm{In}$, Phys. Rev. B 66, 020401 (2002).

[25] B. T. Matthias, A. M. Clogston, H. J. Williams, E. Corenzwit, and R. C. Sherwood, Ferromagnetism in Solid Solutions of Scandium and Indium, Phys. Rev. Lett. 7, 7 (1961).

[26] W. E. Gardner, T. F. Smith, B. W. Howlett, C. W. Chu, and A. Sweedler, Magnetization Measurements and Pressure Dependence of the Curie Point of the Phase $\mathrm{Sc}_{3} \mathrm{In}$, Phys. Rev. 166, 577 (1968).

[27] K. Ikeda and K. A. Gschneider, Disappearance of the Heat Capacity Peak of $\mathrm{Sc}_{3}$ In around the Curie Temperature in High Magnetic Fields, J. Magn. Magn. Mater. 22, 207 (1981). 
[28] J. Takeuchi and Y. Masuda, Low Temperature Specific Heat of Itinerant Electron Ferromagnet $\mathrm{Sc}_{3} \mathrm{In}$, J. Phys. Soc. Jpn. 46, 468 (1979).

[29] J. Grewe, J. S. Schilling, K. Ikeda, and K. A. Gschneidner, Anomalous Behavior of the Weak Itinerant Ferromagnet $\mathrm{Sc}_{3}$ In under Hydrostatic Pressure, Phys. Rev. B 40, 9017 (1989).

[30] R. D. Shannon, Revised Effective Ionic Radii and Systematic Studies of Interatomic Distances in Halides and Chalcogenides, Acta Crystallogr. Sect. A 32, 751 (1976).

[31] V. B. Compton and B. T. Matthias, The Crystal Structure of $\mathrm{Sc}_{3}$ In, Acta Crystallogr. 15, 94 (1962).

[32] T. Jeong and Y. Kwon, Magnetism and Electronic Structure of $\mathrm{Sc}_{3.1} \mathrm{In}$, J. Korean Phys. Soc. 51, 629 (2007).

[33] E. Svanidze, T. Besara, T. Siegrist, K. Han, and E. Morosan, $\mathrm{Ti}_{1-x} \mathrm{Au}_{x}$ Alloys: Hard Biocompatible Metals and Their Possible Applications, arXiv:1103.0095v1.

[34] A. Schenck, Muon Spin Rotation Spectroscopy Principles and Applications in Solid State Physics (Taylor and Francis, New York, 1985).

[35] S. L. Lee, S. H. Kilcoyne, and R. Cywinski, Muon Science: Muons in Physics, Chemistry and Materials (Taylor and Francis, New York, 1999).

[36] R. H. Heffner and K. Nagamine, Special Issue on $\mu \mathrm{SR}$ : Muon Spin Rotation, Relaxation or Resonance, J. Phys. Condens. Matter 16, 4403 (2004).

[37] A. Yaouanc and P. Dalmas de Reotier, Muon Spin Rotation, Relaxation, and Resonance: Applications to Condensed Matter (Oxford University Press, New York, 2010).

[38] J.E. Sonier, MuSR brochure, http://musr.ca/intro/musr/ muSRBrochure.pdf.

[39] T. Moriya, Spin Fluctuations in Itinerant Electron Magnetism (Springer-Verlag, Berlin, 1985).

[40] A. Arrott, Criterion for Ferromagnetism from Observations of Magnetic Isotherms, Phys. Rev. 108, 1394 (1957).

[41] A. Arrott and J. E. Noakes, Approximate Equation of State for Nickel near Its Critical Temperature, Phys. Rev. Lett. 19, 786 (1967).

[42] E. D. Bauer, V. S. Zapf, P.-C. Ho, N. P. Butch, E. J. Freeman, C. Sirvent, and M. B. Maple, Non-Fermi-Liquid Behavior within the Ferromagnetic Phase in $\mathrm{URu}_{2-x} \mathrm{Re}_{x} \mathrm{Si}_{2}$, Phys. Rev. Lett. 94, 046401 (2005).

[43] A. H. Castro Neto and B. A. Jones, Non-Fermi-Liquid Behavior in $U$ and Ce Alloys: Criticality, Disorder, Dissipation, and Griffiths-McCoy Singularities, Phys. Rev. B 62, 14975 (2000).

[44] R. B. Griffiths, Nonanalytical Behavior above the Critical Point in a Random Ising Ferromagnet, Phys. Rev. Lett. 23, 17 (1969).

[45] E. Svanidze, A. J. Millis, and E. Morosan (work in progress).
[46] R. P. Smith, M. Sutherland, G. G. Lonzarich, S. S. Saxena, N. Kimura, S. Tahashima, M. Nohara, and H. Takagi, Marginal Breakdown of the Fermi-Liquid State on the Border of Metallic Ferromagnetism, Nature (London) 455, 1220 (2008).

[47] C. Pfleiderer, A. Faisst, H. von Löhneysen, S. M. Hayden, and G. G. Lonzarich, Field Dependence of the Specific Heat of Single-Crystalline $\mathrm{ZrZn}_{2}$, J. Magn. Magn. Mater. 226, 258 (2001).

[48] M. Nicklas, M. Brando, G. Knebel, F. Mayr, W. Trinkl, and A. Loidl, Non-Fermi-Liquid Behavior at a Ferromagnetic Quantum Critical Point in $\mathrm{Ni}_{x} \mathrm{Pd}_{1-x}$, Phys. Rev. Lett. 82, 4268 (1999).

[49] S. C. Abrahams, A Neutron Diffraction Study of $\mathrm{ZrZn}_{2}$ at $298 K$ and at $5 \mathrm{~K}$, Zeitschrift Kristallographie 112, 427 (1959).

[50] Y. J. Uemura, T. Goko, I. M. Gat-Malureanu, J. P. Carlo, P. L. Russo, A. T. Savici, A. Aczel, G. J. MacDougall, J. A. Rodriguez, and G. M. Luke, Phase Separation and Suppression of Critical Dynamics at Quantum Transitions of Itinerant Magnets: $M n S i$ and $\left(\mathrm{Sr}_{1-x} \mathrm{Ca}_{x}\right) \mathrm{RuO}_{3}$, Nat. Phys. 3, 29 (2007).

[51] Y. J. Uemura, T. Yamazaki, D. R. Harshman, M. Senba, and E. J. Ansaldo, Muon-Spin Relaxation in AuFe and $\mathrm{CuMn}$ Spin Glasses, Phys. Rev. B 31, 546 (1985).

[52] I. M. Gat-Malureanu, J. P. Carlo, T. Goko, A. Fukaya, T. Ito, P. P. Kyriakou, M. I. Larkin, G. M. Luke, P. L. Russo, A. T. Savici, C. R. Wiebe, K. Yoshimura, and Y. J. Uemura, Muon Spin Relaxation and Susceptibility Measurements of an Itinerant-Electron System $\mathrm{Sr}_{1-x} \mathrm{Ca}_{x} \mathrm{RuO}_{3}$ : Quantum Evolution from Ferromagnet to Paramagnet, Phys. Rev. B 84, 224415 (2011).

[53] J. P. Carlo, T. Goko, I. M. Gat-Malureanu, P. L. Russo, A. T. Savici, A. A. Aczel, G. J. MacDougall, J. A. Rodrigues, T. J. Williams, G. M. Luke, C. R. Wiebe, Y. Yoshida, S. Nakatsuji, Y. Maeno, T. Taniguchi, and Y. J. Uemura, New Magnetic Phase Diagram of $(\mathrm{Sr}, \mathrm{Ca})_{2} \mathrm{RuO}_{4}$, Nat. Mater. 11, 323 (2012).

[54] Y. Nozaki, K. Nakanl, T. Yajima, H. Kageyama, B. Frandsen, L. Liu, S. Cheung, T. Goko, Y. J. Uemura, T. S. J. Munsie, T. Medina, G. M. Luke, J. Munevar, D. Nishio-Hamane, and C. M. Brown, Muon Spin Relaxation and Electron/Neutron Diffraction Studies of $\mathrm{BaTi}_{2}\left(\mathrm{As}_{1 x} \mathrm{Sb}_{x}\right)_{2} \mathrm{O}$ : Absence of Static Magnetism and Superlattice Reflections, Phys. Rev. B 88, 214506 (2013).

[55] R. S. Hayano, Y. J. Uemura, J. Imazato, N. Nishida, T. Yamazaki, and R. Kubo, Zero- and Low-Field Spin Relaxation Studied by Positive Muons, Phys. Rev. B 20, 850 (1979). 